\title{
Human iPS cell derived neural cell sheets exhibit mature neural and extendable scaffold functions and promote recovery in injured mouse spinal cords
}

Nagisa Arimitsu1\#, Jun Shimizu ${ }^{1 \#}$, Masahiro Iinuma $^{2 \#}$, Tasuku Umehara ${ }^{2}$, Naruyoshi Fujiwara ${ }^{1}$, Kenji Takai $^{1}$, Yuji Ueda ${ }^{1}$, Sueshige Wakisaka $^{1}$, Chieko Hirotsu ${ }^{1}$, Tomoko Suzuki ${ }^{1}$, Moroe Beppu ${ }^{2}$, Hisateru Niki $^{2}$ and Noboru Suzuki ${ }^{1}$

${ }^{1}$ Department of Immunology and Medicine, St. Marianna University School of Medicine, Japan

${ }^{2}$ Department of Orthopedics, St. Marianna University School of Medicine, Japan

\#These authors contributed equally to the work

\begin{abstract}
We developed neural cell sheets being positive for mature motoneuron markers, such as $\beta \mathrm{III}$ tubulin, islet1 and HB9, from human induced pluripotent stem (hiPS) cells after embryoid body formation. We transplanted the neural cell sheets into the complete transection sites of spinal cord injury (SCI) model mice. We assessed functional and histological analyses of the SCI mice and compared the data with those of hiPS cell derived neural stem/progenitor (NSP) cell transplanted SCI mice.

Neurofilament medium (NFM), $\beta$ III tubulin, and HB9 positive cells were significantly increased in vitro in neural cell sheets compared with those in NSP cells. The neural cell sheets expressed remarkably high mRNA of NFM and islet 1 compared with NSP cells. After transplantation, the neural cell sheets elongated human neural cell adhesion molecule (hNCAM) positive axons over the L1 spinal level and carried a neuron tracer cholera toxin $\beta$ subunit (CT $\beta$ ) to downstream region. The sheet transplantation showed significantly better locomotor recovery of SCI mice until day 65 than NSP cell transplantation. The sheet transplantation tended to improve spinal cord atrophy in caudal part of injured/grafted site to the same extent as NSP cell transplantation. We suggest that the neural cell sheets derived from hiPS cells may be one of the most effective treatment for SCI, through the mature neural and extendable scaffold functions.
\end{abstract}

\section{Introduction}

Stem cell replacement therapy has been shown to be useful for the treatment of patients with spinal cord injury (SCI) in several clinical trials using autologous bone marrow stem cells [1], and mesenchymal stem cells [2]. It was suggested that transplanted NSP cells replaced injured neurons, restored disrupted neural circuits, and remyelinated axons in patients with SCI $[1,3,4]$.

Human induced pluripotent stem (hiPS) cells are becoming one of the promising candidates for a new cell source of transplantation without ethical problems and immune rejection because it is possible to establish human "individual specific" iPS cells from patient's somatic cells [5].

We previously demonstrated that motoneurons were successfully induced from hiPS cells using retinoic acid (RA), noggin (NOG) and sonic hedgehog (SHH) [6].

The neurons were functional in vivo even in the complete transection sites of SCI model mice and brought about motor functional recovery of hind limbs of the mice [7].

Here, we investigated a new cell engineering technique for formation of neural cell sheets using temperature-responsive gelation polymer coated dishes $\left(\mathrm{Upcell}^{\circledR}\right)$ to adapt the grafts effectively to the complete transection sites of SCI model mice. The new technique allows us to obtain mature and highly stable neural cell sheets. We expected that the mature neural cell sheets worked as effective replacement for injured mouse cells with the extendable scaffold function.
The neural cell sheets expressed $\beta$ III tubulin, islet1, HB9, which are defined as mature neuron and motoneuron markers. We transplanted the neural cell sheets into the complete transection sites of SCI model mice and compared the behavioral test scores with those of neural stem/progenitor (NSP) cell transplantation.

\section{Materials and methods}

\section{Induction of neural differentiation of hiPS cells}

The hiPS cell lines (253G1 and 201B7, RIKEN, Tsukuba, Japan) were used in this study. Both cell lines gave essentially the same results, thus we presented the results obtained using 253G1 cell line.

Undifferentiated hiPS cells were maintained in growth medium consisting of DMEM/F12 supplemented with non-essential amino acids, pyruvate, $0.1 \mathrm{mM} \beta$-mercaptoethanol, $2 \mathrm{mM}$ L-glutamine (all purchased from Gibco, Grand Island, NY), 20\% knockout serum replacement (KSR, Gibco) and basic fibroblast growth factor (bFGF, $\mathrm{R} \& \mathrm{D}$ systems, Minneapolis, MN). The cells were cultured on mitomycin

Correspondence to: Noboru Suzuki MD, Ph.D, Department of Immunology and Medicine, St. Marianna University School of Medicine, 2-16-1 Sugao, Miyamaeku, Kawasaki, Kanagawa 216-8511, Japan, Tel: +81-44-977-8111 (ex 3547), Fax: +81-44-976-3315, E-mail: n3suzuki@marianna-u.ac.jp

Key words: human iPS cells, neural cell sheet, spinal cord injury, spinal motor neuron, transplantation

Received: June 02, 2016; Accepted: June 30, 2016; Published: July 05, 2016 

cords

$\mathrm{C}$ treated mouse emryonic fibroblasts (MEF) as a feeder layer. The schedule for the neural cell induction was shown in Figure 1A. Day 0 was the starting date of embryoid body (EB) formation. We developed EB from undifferentiated hiPS cells in a floating condition for 4 days (from day 0 to day 4). Then the cells were cultured in fibronectin (FN, BD Biosciences, San Diego, CA) coated dishes for 4 days (from day 4 to day 8 ). We introduced $1 \mu \mathrm{M}$ RA (Sigma, Tokyo, Japan), $10 \mathrm{nM}$ NOG (NOG-Fc, R\&D systems) and $10 \mathrm{nM} \mathrm{SHH} \mathrm{(R \& D} \mathrm{systems)} \mathrm{into}$ the culture twice (at day 5 and day 7 ).
We have already tested a variety of culture conditions employing different culture media and growth factors, and found that medium containing the defined amounts of the three factors (RA, NOG and $\mathrm{SHH}$ ) were most suitable for inducing the differentiation into neurons including those with spinal motoneuron phenotype from hiPS cells [7].

\section{Reverse transcription-polymerase chain reaction (RT-PCR)}

Total RNA from cultured cells was isolated using the RNeasy kit (Qiagen, Hilden, Germany). Complementary DNA was synthesized

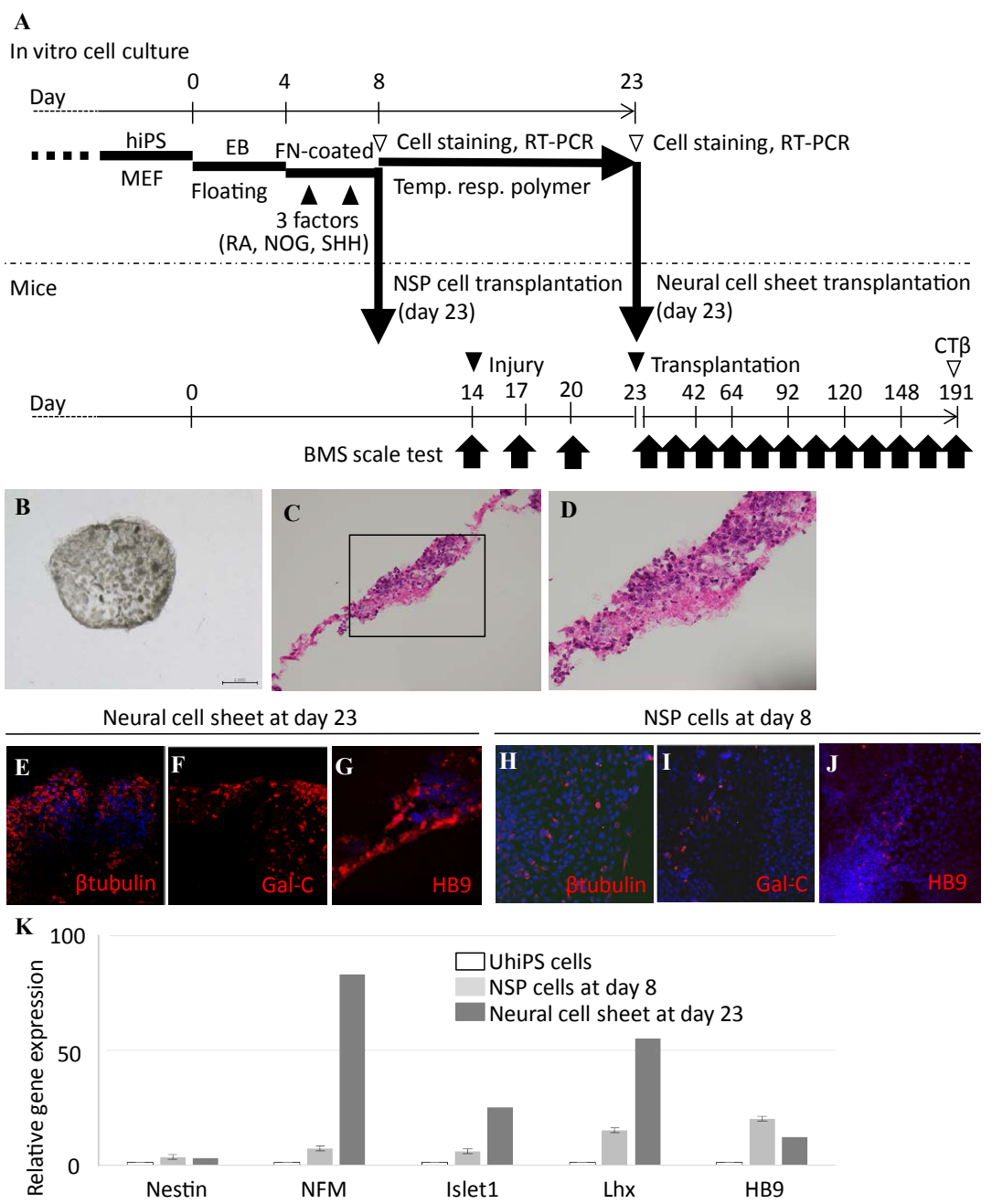

Figure 1. In vitro characterization of neural cell sheets derived from human iPS cells.

A. A schematic representation of neural cell sheet formation and the transplantation. Undifferentiated hiPS cells were maintained in a growth medium on a feeder layer consisting of mouse embryonic fibroblasts (MEF). We developed embryoid body (EB) from undifferentiated hiPS cells in a floating condition (Floating) for 4 days. Then the cells were cultured in fibronectin (FN)-coated dishes for 4 days. We introduced retinoic acid (RA), noggin (NOG), and sonic hedgehog (SHH) (3 factors) into the culture twice (at day 5 and day 7 ). The neural stem/progenitor (NSP) cells were disaggregated into single cell suspensions at day 8 . The NSP cells at day 8 were utilized for NSP cell transplantation, immunocytochemistry, and RT-PCR. An aliquot of the

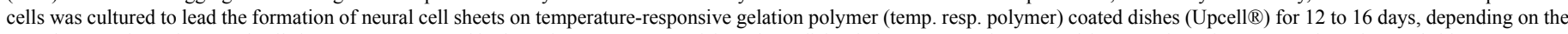
neural maturation. The neural cell sheets were recovered by lowering temperature of the culture dishes below $22^{\circ} \mathrm{C}$, and were used for transplantation. We conducted open-field Basso Mouse Scale (BMS) scoring on the first day, third day, and once every two weeks after injury up to day 191. Spinal cord tissues were obtained from SCI mice for histological analysis at day 191.

B. A stereomicroscopic view of the neural cell sheet cultured in a 48 well culture plate.

C. Hematoxylin and eosin (HE) staining of the neural cell sheet.

D. Higher magnification of panel C inset. The polymer culturing promoted polarization of cell growth in the cell sheet formation, resulting in the differences of cell density by location.

E-G. Immunocytochemical analyses of the neural cell sheet at day 23 .

H-J. Immunocytochemical analyses of the NSP cells at day 8. The neural cell sheets and NSP cells were stained with several mature neuron markers. The marker positive cells were significantly increased in the neural cell sheets compared with those in NSP cells (Table 1). The cells were counterstained with DAPI (blue). Scale bar: $50 \mu \mathrm{m}$.

K. Relative gene expression analyses of neural cell sheets and NSP cells were conducted by quantitative RT-PCR for neuron associated molecules. We observed higher expressions of NFM and Islet1 mRNA in neural cell sheets compared with those in NSP cells.

$\beta$ tubulin: $\beta$ III tubulin, Gal-C: Galactocerebroside, NFM: neurofilament middle chain, Lhx: Lim-homeobox protein, UhiPS: Undifferentiated hiPS. 

cords

with Taq Man reverse transcription reagents (Applied Biosystems, Foster City, CA) using random hexamers as primers, in accordance with the manufacturer's instructions. In quantitative RT-PCR, eukaryotic $18 \mathrm{~S}$ ribosomal RNA was used as an endogenous control. We studied five combinations of TaqMan primers and probes for the following molecules: Nestin, neurofilament medium (NFM), islet1, Lim-homeobox protein (Lhx), and HB9 (all from Applied Biosystems). Relative expression in differentiated cells was calculated by $2^{-\Delta \Delta C t}$ method and was compared to that in undifferentiated cells.

\section{Experimental SCI}

Female C57BL/6 mice (5-12 weeks old, Japan SLC) were used as transplant recipients because we wanted to use genetically normal mice $[7,8]$. Animals were housed less than 5/cage with mouse food and water adlib on a $12 / 12 \mathrm{hr}$ light/dark cycle. Surgical interventions, pre- and postsurgical animal care, and euthanasia followed the Guide for the Care and Use of Laboratory Animals, 8th edition (National Research Council) and were approved by the local Animal Care Committee (Institutional Animal Care and Use Committee).

The mice were anesthetized with an intraperitoneal injection of 40$50 \mathrm{mg} / \mathrm{kg}$ pentobarbital (Merck Animal Health, Summit, NJ) and 10 $\mathrm{mg} / \mathrm{kg}$ xylazine (Bayel AG, Leverkusen, Germany). Laminectomy was performed at spinal level Th11. We slit the dura matter and exposed the spinal cord. We completely transected the entire depth of the spinal cord using No. 11 scalpel blade and probed the vertebral cavity several times with a fine spatula to ensure the complete spinal cord transection. After the injury, muscle layers over the laminectomy and skin on the back were sutured.

\section{Cell sheet generation}

The cells were cultured on temperature-responsive gelation polymer $[9,10]$ coated dishes (Upcell ${ }^{\circledR}$, CellSeed, Tokyo, Japan) for 12 to 16 days (average 15 days) depending on the neural maturation, where they extended axon-like processes, leading to the formation of neural cell sheet. The polymer had the reversible sol-gel process by temperature to recover the cell sheets flawlessly. Before transplantation, the polymer coated dishes were placed on $22^{\circ} \mathrm{C}$ incubator for 1 hour for the recovery.

\section{Neural cell sheet transplantation}

Neural cell sheets, NSP cells, and vehicle (PBS) were placed into the transection sites and covered with the temperature-responsive gelation polymer to retain the grafts $[7,9,10]$. The polymer was then covered with a cement protector before the suture of muscle layers. All mouse groups (two transplantation groups and PBS injection group) were administered $10 \mathrm{mg} / \mathrm{Kg}$ cyclosporine (Novartis Pharmaceuticals Japan, Tokyo, Japan) by subcutaneous injection, and $0.2 \mathrm{mg} / \mathrm{Kg}$ dexamethasone (Sigma) by intraperitoneal injection one hour before the transplantation. $10 \mathrm{mg} / \mathrm{Kg}$ cyclosporine was given into all mice once a day from the next day of the transplantation until the mice were sacrificed.

\section{Behavioral analysis}

Functional recovery of mice after SCI was evaluated by openfield Basso Mouse Scale (BMS) [11]. We conducted the BMS scoring experiments on the first day, third day and once per two weeks after injury (Figure 1). We compared the mean scores of both treatment groups for up to day 191.

\section{Immunofluorescence staining}

Immunofluorescence staining was conducted as reported previously [6-8]. In brief, for all immunofluorescence procedures, consecutive tissue slices were made for immunohistochemistry of neuron associated proteins. For in vitro cell culture assay, cultured neurons were fixed in $4 \%$ paraformaldehyde (Merck, Darmstadt, Germany) for 15 minutes after PBS washing. Cells and $20 \mu \mathrm{m}$ thick cryostat (CM1850, Leica, Wetzlar, Germany) sections were blocked for 2 hours in PBSTG, which was contained $0.2 \%$ Tween 20 (Kanto Chemical, Tokyo, Japan) and 5\% goat serum (Gibco) in PBS, with or without a mouse on mouse blocking reagent (Vector Laboratories, Burlingame, $\mathrm{CA})$. The sections were incubated overnight with appropriate primary antibodies. The fixed cells and brain tissues were stained with rat antiNestin (Millipore, Billerica, MA) (1:400), rabbit anti-NFM (Millipore) (1:200), mouse anti-ßIII tubulin (Promega, Madison, WI) (1:400), mouse anti-HB9 (Developmental Studies Hybridoma Bank, Iowa City, IA) (1:100), mouse anti-human Nuclei (hNuc, Abnova, Taipei City, Taiwan) (1:100), rabbit anti-Glial fibrillary acidic protein (GFAP, Dako, Glostrup, Denmark) (1:1000), mouse anti-Galactocerebroside (Gal-C, Millipore) (1:100), mouse anti-Synaptophysin (Dako) (1:100), and mouse anti-human Neural Cell Adhesion Molecule (hNCAM, Beckman Coulter, Brea, CA) (1:100) antibodies. We used anti-hNuc and anti-hNCAM antibodies to detect human neurons in the recipient mouse spinal cords. As a control staining, adjacent tissue samples were stained essentially with the same procedure without addition of the primary antibodies. In the immunocytochemistry, we counted at least 200 cells in each experiment to calculate the number of cells expressing neural cell associated proteins in vitro. Each percentage of the antigen positive cells in immunofluorescence study was expressed as mean and standard error of the mean (s. e. m.) of at least three independent experiments $(n \geq 3)$.

We stained the cryostat sections with hematoxylin-eosin (HE, Wako, Osaka, Japan) to observe cell localization and morphological features.

\section{Anterograde tracing of the injured spinal cord after neural cell sheet transplantation}

We put a small laminectomy at Th10 of spine in the SCI mice. We injected $2 \mu$ of $1 \%$ Alexa Fluor ${ }^{\circledR} 594$ conjugated cholera toxin $\beta$-subunit (CT $\beta$, Life Technologies, Carlsbad, CA) $[7,12]$ in PBS into spinal cord using a glass capillary. 24 hours after the injection, mice were sacrificed and spinal cords were processed for fluorescence analyses.

\section{Statistical analysis}

Significance of difference between two groups was determined by using Mann-Whitney tests. Continuous variables throughout 191 days subjected to repeated measurements over a period of time on the BMS scoring were analyzed using a repeated measures multivariate analysis of variance (MANOVA) followed by Tukey's post-test using addin soft $\mathrm{XLSTAT}^{\circledR}$ 3.02. A $p$ value less than 0.05 was considered significant.

\section{Results}

\section{Assessments of motoneuron associated proteins and genes in neural cell sheets and NSP cells}

We cultured the aliquot of NSP cells in dishes coated with temperature-responsive gelation polymers for 10-16 days. We obtained intact cell sheets by lowering temperature of culture dishes below $22^{\circ} \mathrm{C}$ (Figure 1B). HE staining of the sheets showed that the 

cords

polymer culturing promoted polarization of cell growth in the cell sheet formation, resulting in the differences of cell density by location (Figures 1C, 1D).

Mature motoneuron related protein positive cells were significantly increased in the neural cell sheets compared with those in NSP cells (Table 1). The neural cell sheets expressed $\beta I I I$ tubulin and the percentage of $\beta I I I$ tubulin positive cells was $83 \pm 5.7 \%$ (Figure $1 \mathrm{E})$. The NSP cells expressed $\beta \mathrm{III}$ tubulin and the percentage of $\beta \mathrm{III}$ tubulin positive cells was $25 \pm 1.8 \%$ (Figure $1 \mathrm{H}$ ). The neural cell sheets expressed Gal-C and the percentage of Gal-C positive cells was $25 \pm$ $5.4 \%$ (Figure 1F). The NSP cells expressed Gal-C and the percentage of Gal-C positive cells was $6.4 \pm 1.9 \%$ (Figure 1I). The neural cell sheets expressed HB9 and the percentage of HB9 positive cells was $30 \pm 5.5 \%$ (Figure 1G). The NSP cells expressed HB9 and the percentage of HB9 positive cells was $9.1 \pm 3.8 \%$ (Figure $1 \mathrm{~J}$ ).

We conducted quantitative RT-PCR of Nestin, NFM, Islet1, Lhx and HB9 in neural cell sheets and NSP cells. Relative gene expressions of NFM, Islet1, and Lhx in neural cell sheets were remarkably high compared with those of NSP cells (Figure 1K).

\section{Behavioral analysis}

Functional recovery of mice after SCI was evaluated by BMS scoring up to day 191. Motor functions of neural cell sheet transplanted mice and NSP cell transplanted mice were significantly improved after day 37 compared with that of vehicle injected mice (single asterisks of Figure 2). The sheet transplantation significantly improved the locomotor functions of SCI mice until day 65 compared with NSP cell transplantation (a double asterisk of Figure 2).

Histological and immunohistochemical analyses of the injured spinal cords after neural cell sheet transplantation

Mouse spinal cords were processed for histopathological analyses 168 days after the transplantation. It looked that the neural cell sheets and NSP cells successfully connected the transected spinal cords in HE staining (Figures 3A1, 3A2). We observed that neural cell sheet and NSP cell transplantation tended to improve the atrophy and gliosis of host spinal cord compared with vehicle injection (Figures 3A1, 3A2).
The graft neural function and stability were evaluated by immunohistochemistry for NFM, GFAP, HB9, Gal-C, $\beta I I I$ tubulin, and synaptophysin. We found hNuc and NFM positive human neurons locating diffusely in the transection site of a neural cell sheet transplanted SCI mouse and an NSP cell transplanted SCI mouse (Figures 3B1-3B4). HB9 positive neurons were found in the grafted and scarring areas of a sheet transplanted SCI mouse (Figures $3 \mathrm{C} 1,3 \mathrm{C} 2$ ). GFAP expression reduced remarkably in a neural cell sheet transplanted SCI mouse compared with those in a PBS injected SCI mouse (Figures $3 \mathrm{C} 1,3 \mathrm{C} 2,3 \mathrm{C} 5,3 \mathrm{C} 6)$. We found that $\beta \mathrm{III}$ tubulin positive neurons and Gal-C positive cells were abundant in a neural cell sheet transplanted SCI mouse compared with those in a PBS injected SCI mouse (FigureS 3D1,3D2,3D5,3D6,3E1,3E2,3E5,3E6). Synaptophysin expressions were widely observed in a neural cell sheet transplanted SCI mouse and an NSP cell transplanted SCI mouse compared with that in PBS injected SCI mice (Figure 3F1-3F6).

\section{Anterograde tracing of the injured spinal cords after neural cell sheet transplantation}

We conducted anterograde tracing experiments of the injured spinal cords using CT $\beta$ for detection of the tracer labeled axons in caudal sites of the lesion 168 days after the transplantation (Figure 4A). The injured spinal cord was injected with CT $\beta$ at the spinal level Th10 (Figure 4A). 24 hours later, we conducted immunohistochemistry for $\beta I I I$ tubulin and hNCAM using adjacent sections of the injured spinal cords. We examined CT $\beta$ expressions around the spinal level L1 (Figure 4A). We did not observe CT $\beta$-labeled axons in the injured spinal cords of PBS injected SCI mice (Figures $4 \mathrm{~B}, 4 \mathrm{C}$ ). We found $\beta \mathrm{III}$ tubulin and CT $\beta$ double positive axons at the spinal level L1 (arrow heads of Figure $4 \mathrm{D}$ ). Similarly, hNCAM and CT $\beta$ positive axons were identified at the spinal level L1 (arrow heads of Figure 4E).

\section{Discussion}

We successfully generated mature neural cell sheets using temperature-responsive gelation polymer coated dishes $\left(\mathrm{Upcell}^{\mathbb{\mathbb { }}}{ }^{\mathrm{B}}\right.$. Development of neural cell sheets using the tissue engineering approach enabled us to effectively provide the neurons into the lesion without artificial materials. We compared the motoneuron functional

\section{BMS score}

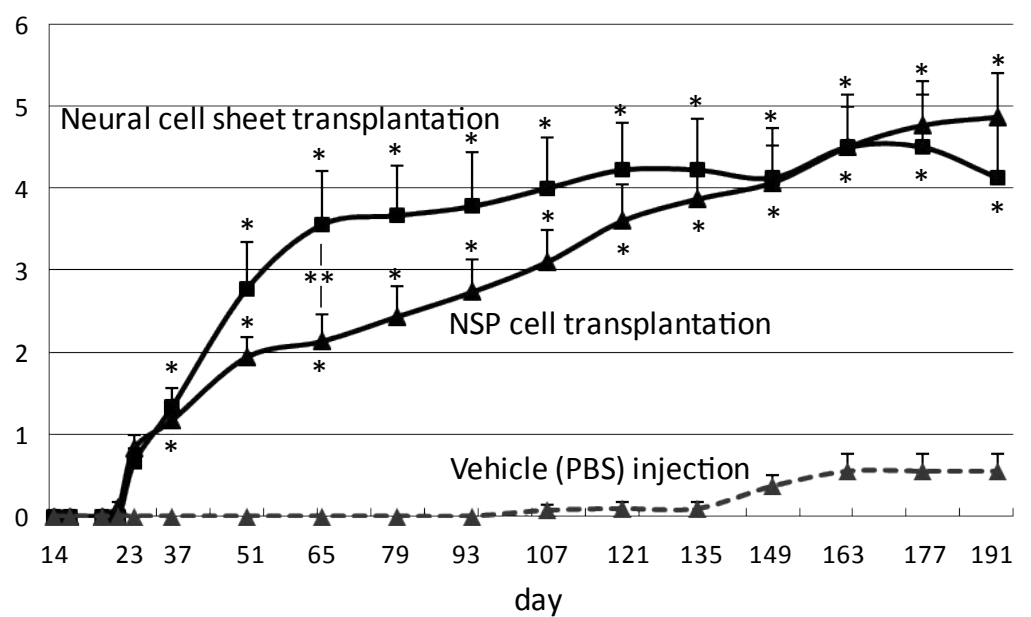

Figure 2. Behavioral analysis of SCI mice with neural sheet transplantation.

Functional recovery of mice after SCI was evaluated by BMS scoring up to day 191 . Motor functions of neural cell sheet transplanted mice and NSP cell transplanted mice were significantly improved after day 37 compared with that of vehicle injected mice (single asterisks). The sheet transplantation significantly improved the locomotor function of SCI mice until day 65 compared with NSP cell transplantation (a double asterisk). 

cords

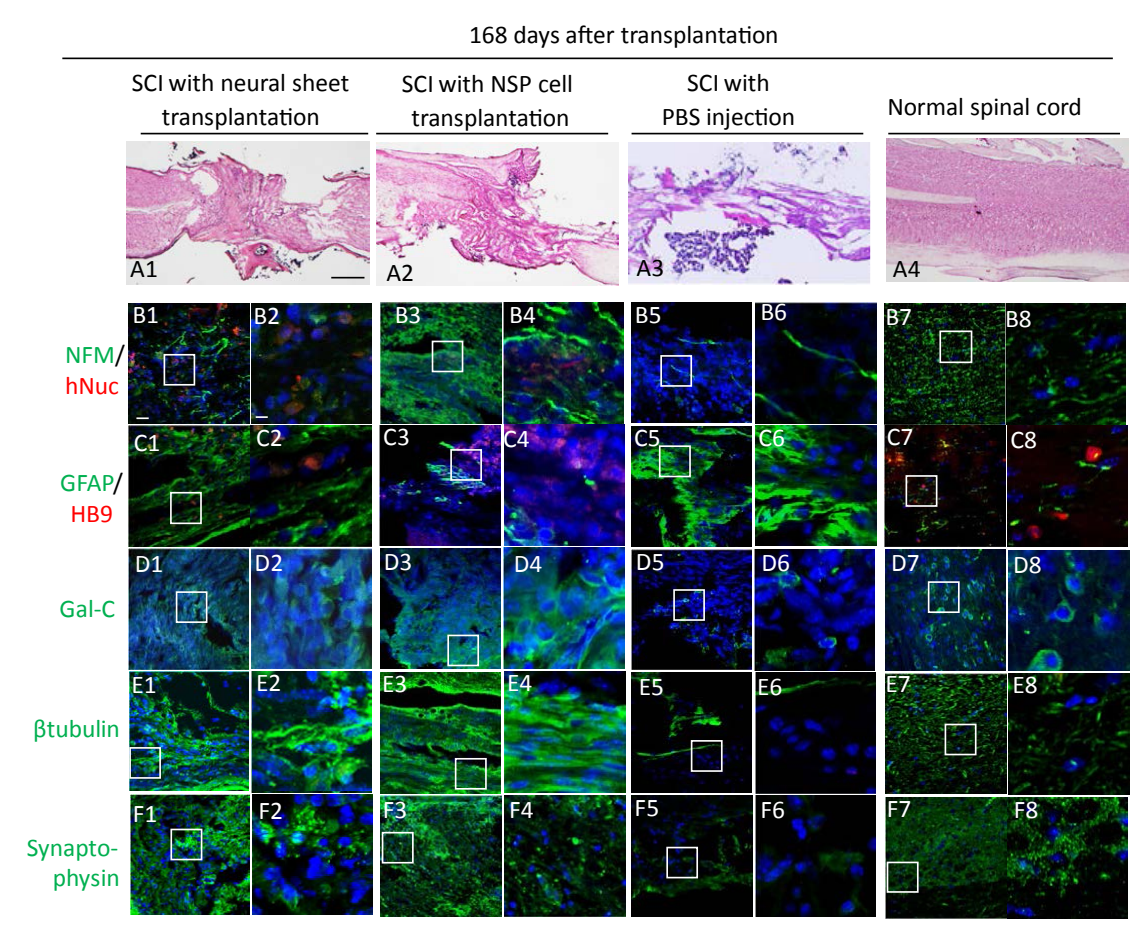

Figure 3. Histological and immunohistochemical analyses of the injured spinal cords after neural cell sheet transplantation.

A. Mouse spinal cords were processed for histological analyses 168 days after the transplantation by hematoxylin and eosin (HE) staining of longitudinal sections.

A1-A2. HE staining of the spinal cords after neural cell sheet transplantation (A1) and NSP cell transplantation (A2). We observed that the transected sites were fulfilled with a large number of cells, suggesting grafted cells survived in the lesion. The gliosis of the spinal cords was remarkably reduced in the tissues of two transplanted groups compared with that of vehicle injected mice. A3. HE staining of the spinal cord after vehicle injection. A large cavity and gliosis of spinal cord were found.

A4. HE staining of the normal spinal cord.

B-F. We analyzed the expressions of neuron associated proteins in the spinal cords 168 days after the transplantation by immunofluorescence with DAPI nuclear staining (blue). Scale bar: $20 \mu \mathrm{m}$.

B1. Anti-human Nuclei (hNuc) staining (red) and anti-Neurofilament middle chain (NFM) staining (green) of the injured spinal cord with neural cell sheet transplantation.

B2. Higher magnification of panel B1 inset. Scale bar: $5 \mu \mathrm{m}$.

B3. Anti-hNuc staining (red) and anti-NFM staining (green) of the injured spinal cord with NSP cell transplantation.

B4. Higher magnification of panel B3 inset.

B5. Anti-hNuc staining (red) and anti-NFM staining (green) of the injured spinal cord with PBS injection.

B6. Higher magnification of panel B5 inset.

B7. Anti-hNuc staining (red) and anti-NFM staining (green) of the normal spinal cord.

B8. Higher magnification of panel B7 inset.

C1. Anti-HB9 staining (red) and anti-Glial fibrillary acidic protein (GFAP) staining (green) of the injured spinal cord with neural cell sheet transplantation.

C2. Higher magnification of panel $\mathrm{C} 1$ inset.

C3. Anti-HB9 staining (red) and anti-GFAP staining (green) of the injured spinal cord with NSP cell transplantation.

C4. Higher magnification of panel $\mathrm{C} 3$ inset.

C5. Anti-HB9 staining (red) and anti-GFAP staining (green) of the injured spinal cord with PBS injection.

C6. Higher magnification of panel C5 inset.

C7. Anti-HB9 staining (red) and anti-GFAP staining (green) of the normal spinal cord.

C8. Higher magnification of panel $\mathrm{C} 7$ inset.

D1. Anti-Galactocerebroside (Gal-C) staining (green) of the injured spinal cord with neural cell sheet transplantation.

D2. Higher magnification of panel D1 inset.

D3. Anti-Gal-C staining (green) of the injured spinal cord with NSP cell transplantation.

D4. Higher magnification of panel D3 inset.

D5. Anti-Gal-C staining (green) of the injured spinal cord with PBS injection.

D6. Higher magnification of panel D5 inset.

D7. Anti-Gal-C staining (green) of the normal spinal cord.

D8. Higher magnification of panel D7 inset.

E1. Anti- $\beta$ III tubulin ( $\beta$ tubulin) staining (green) of the injured spinal cord with neural cell sheet transplantation.

E2. Higher magnification of panel E1 inset.

E3. Anti- $\beta I I I$ tubulin staining (green) of the injured spinal cord with neuron transplantation.

E4. Higher magnification of panel E3 inset.

E5. Anti-BIII tubulin staining (green) of the injured spinal cord with PBS injection.

E6. Higher magnification of panel E5 inset.

E7. Anti- $\beta$ III tubulin staining (green) of the normal spinal cord.

E8. Higher magnification of panel E7 inset.

F1. Anti-Synaptophysin staining (green) of the injured spinal cord with neural cell sheet transplantation.

F2. Higher magnification of panel F1 inset.

F3. Anti-Synaptophysin staining (green) of the injured spinal cord with NSP cell transplantation.

F4. Higher magnification of panel F3 inset.

F5. Anti-Synaptophysin staining (green) of the injured spinal cord with PBS injection.

F6. Higher magnification of panel F5 inset.

F7. Anti-Synaptophysin staining (green) of the normal spinal cord.

F8. Higher magnification of panel F7 inset.

We found hNuc and NFM positive human neurons locating diffusely in the transection site of a neural cell sheet transplanted SCI mouse and an NSP cell transplanted SCI mouse (B1-B4). HB9 positive neurons were found in the grafted and scarring areas of a sheet transplanted SCI mouse $(\mathrm{C} 1, \mathrm{C} 2)$. GFAP expression reduced remarkably in a neural cell sheet transplanted SCI mouse compared with those in a PBS injected SCI mouse $(\mathrm{C} 1, \mathrm{C} 2, \mathrm{C} 5, \mathrm{C} 6)$. We found that $\beta \mathrm{III}$ tubulin positive neurons and Gal-C positive cells were abundant in a neural cell sheet transplanted SCI mouse compared with those in a PBS injected SCI mouse (D1, D2, D5, D6, E1, E2, E5, E6). Synaptophysin expressions were widely observed in a neural cell sheet transplanted SCI mouse and an NSP cell transplanted SCI mouse compared with that in PBS injected SCI mice (F1-F6). 

cords

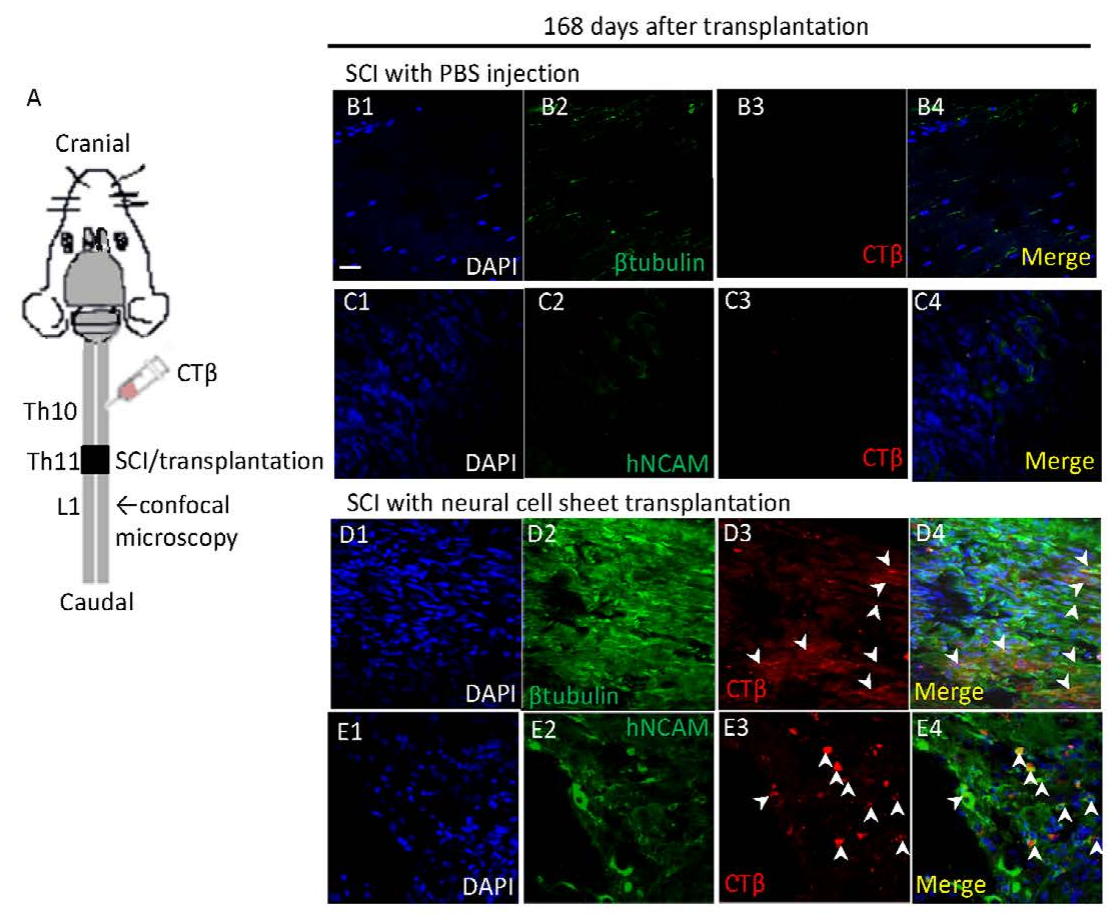

Figure 4. Anterograde tracing of the injured spinal cords after neural cell sheet transplantation.

We conducted anterograde tracing of the injured spinal cord using CT $\beta 168$ days after the transplantation.

A. A schematic representation of CT $\beta$ tracing experiment in mice. We injected CT $\beta$ at the spinal level Th10 (complete transection was performed at the spinal level Th11). 24 hours later, we conducted immunohistochemistry for $\beta$ III tubulin ( $\beta$ tubulin) and human specific NCAM (hNCAM) using adjacent sections of the injured spinal cords. We examined the CT $\beta$ expression around the spinal level L1.

B-E. Immunohistochemistry of the injured spinal cords after vehicle injection (B-C) and neural cell sheet transplantation (D-E).

B1-B4. Anti- $\beta$ III tubulin staining (green) and CT $\beta$ expression (red). Scale bar: $20 \mu \mathrm{m}$.

B1. DAPI staining (blue).

B2. Anti- $\beta$ III tubulin staining.

B3. CT $\beta$ expression.

B4. Merged image of B1, B2, and B3.

C1-C4. Anti-hNCAM staining (green) and CT $\beta$ expression (red)

C1. DAPI staining (blue).

C2. Anti-hNCAM staining

C3. CT $\beta$ expression.

C4. Merged image of $\mathrm{C} 1, \mathrm{C} 2$, and $\mathrm{C} 3$.

D1-D4. Anti- $\beta$ III tubulin staining (green) and CT $\beta$ expression (red).

D1. DAPI staining (blue).

D2. Anti- $\beta$ III tubulin staining.

D3. CT $\beta$ expression.

D4. Merged image of D1, D2, and D3. We found $\beta I I I$ tubulin and CT $\beta$ double positive axons at the spinal level L1 (arrow heads).

E1-E4. Anti-hNCAM staining (green) and CT $\beta$ expression (red).

E1. DAPI staining (blue).

E2. Anti-hNCAM staining.

E3. CT $\beta$ expression.

E4. Merged image of E1, E2, and E3. We found hNCAM and CT $\beta$ double positive axons at the spinal level L1 (arrow heads).

recovery between neural cell sheet transplantation and NSP cell transplantation. The neural cell sheet transplantation significantly improved the analytical behavioral scores until day 65 compared with NSP cell transplantation. The beneficial effects of neural cell sheets on the motoneuron function gradually decreased and the analytical behavioral scores became comparable between the two transplantation groups throughout the observation period (191 days).

We induced NSP cells enriched with motoneurons in vitro from hiPS cells using RA, NOG, and SHH [7]. In vitro characterization showed that the neural cell sheets expressed at significantly higher levels of mature motoneuron markers, namely $\beta$ tubulin and HB9, than NSP cells (Figure 1E, 1G, 1H, 1J, and Table 1). The neural cell sheets remarkably increased gene expressions of NFM, Islet1, and Lhx compared with NSP cells (Figure 1K). We suggest that the neural cell
Table 1. In vitro characterization of mature motoneuron associated protein expressions in neural cell sheets and $\mathrm{NSP}^{\mathrm{a}}$ cells $\mathrm{s}^{\mathrm{b}}$.

\begin{tabular}{|c|c|c|c|}
\hline & Neural cell sheets (\%) & NSP cells (\%) & p-value $^{\mathbf{c}}$ \\
\hline BIII tubulin & $83 \pm 5.7$ & $25 \pm 1.8$ & $<0.05$ \\
\hline Gal-C & $25 \pm 5.4$ & $6.4 \pm 1.9$ & $<0.05$ \\
\hline HB9 & $30 \pm 5.5$ & $9.1 \pm 3.8$ & $<0.05$ \\
\hline
\end{tabular}

aNSP: neural stem/progenitor. ${ }^{b}$ All data are expressed as mean \pm s.e.m from at least three independent experiments in each group. ${ }^{~} \mathrm{P}$ values were calculated by Mann-Whitney tests. ${ }^{\mathrm{d}} \mathrm{Gal}-\mathrm{C}$ : Galactocerebroside.

sheets may remain in a state of readiness for the transplantation with the mature neural and extendable scaffold functions.

Histopathological analyses of SCI mice showed that HB9 expressing cells were more prevalent in the spine transection sites of neural cell sheet transplanted mice than in those of NSP cell transplanted mice 

cords

(Figure 4C). We observed that Gal-C expressing cells increased and GFAP expressing cells deceased in neural cell sheet and NPG cell transplanted mice compared with PBS as vehicle injected mice (Figure 4C, 4D). These results suggest that the neurons grafted into the transected sites may exert long-term inhibitory effects on the scar formation [13].

СT $\beta$ is an anterograde and retrograde tracer and is absorbed and transported by a strictly limited group of damaged neurons whose body and axons were located around the injected site [14]. We injected CT $\beta$ into Th10 of spinal cord, a cranial site of injury/transplantation. It was carried down to caudal spinal cord beyond the lesion of Th11. It reached L1 of detection site on $\beta$ III tubulin and hNCAM positive axons (Figure 4D, 4E). We revealed that majority of CT $\beta$ positive axons simultaneously expressed hNCAM in the spinal cords grafted with neural cell sheets (Figure 4E). We suggest that damaged host neurons may transport CT $\beta$ to the transection site and transplanted neurons of neural cell sheets may absorb the CT $\beta$ and transport further to the caudal spinal cord.

In conclusion, our findings suggest that both hiPS cell derived neural cell sheet transplantation and NSP cell transplantation may be powerful tools for the treatment of patients with severe SCI. It may be possible to utilize both types of cells, for example, initially grafted with neural cell sheets for the space reservations and followed by repetitive transplantation of NSP cells, for maximizing the benefit of the stem cell therapy.

\section{References}

1. Mothe AJ, Tator CH (2012) Advances in stem cell therapy for spinal cord injury. J Clin Invest 122: 3824-3834. [Crossref]

2. Park JH, Kim DY, Sung IY, Choi GH, Jeon MH, et al. (2012) Long-term results of spinal cord injury therapy using mesenchymal stem cells derived from bone marrow in humans. Neurosurgery 70: 1238-1247.[Crossref]

3. Abematsu M, Tsujimura K, Yamano M Saito M, Kohno K, et al. (2010) Neurons derived from transplanted neural stem cells restore disrupted neural circuitry in a mouse model of spinal cord injury. J Clin Invest 120: 3255-3266.[Crossref]
4. Kobayashi Y, Okada Y, Itakura G, Iwai H, Nishimura S, et al. (2012) Pre-evaluated safe human iPSC-derived neural stem cells promote functional recovery after spinal cord injury in common marmoset without tumorigenicity. PLoS One 7: e52787.[Crossref]

5. Takahashi K, Tanabe K, Ohnuki M, Narita M, Ichisaka T et al. (2007) Induction of pluripotent stem cells from adult human fibroblasts by defined factors. Cell 131: 861872.[Crossref]

6. Fujii A, Chiba S, Takada E, Ueda Y, Shimizu J, et al. (2009) Generation of spinal motoneurons from mouse induced pluripotent stem cells. St. Marianna Medical J 37: 327-336.[Crossref]

7. Iinuma M, Umehara T, Arimitsu N, Shimizu J, Misawa H, et al. (2015) Induction of neural cells with spinal motoneuron phenotype from human iPS cells and the transplantation to totally transected spinal cords in mice. Inflammation and Regeneration 3: 154-163.[Crossref]

8. Hamada M, Yoshikawa H, Ueda Y, Kurokawa MS, Watanabe K, et al. (2006) Introduction of the MASH1 gene into mouse embryonic stem cells leads to differentiation of motoneuron precursors lacking Nogo receptor expression that can be applicable for transplantation to spinal cord injury. Neurobiol Dis 22: 509-522. [Crossref]

9. Nagaya M, Kubota S, Suzuki N, Akashi K, Mitaka T (2006) Thermoreversible gelation polymer induces the emergence of hepatic stem cells in the partially injured rat liver. Hepatology 43: 1053-1062.[Crossref]

10. Nagaya M, Kubota S, Suzuki N, Tadokoro M, Akashi K (2004) Evaluation of thermoreversible gelation polymer for regeneration of focal liver injury. EurSurg Res 36: 95-103.[Crossref]

11. Basso DM, Fisher LC, Anderson AJ, Jakeman LB, McTigue DM, et al. (2006) Basso Mouse Scale for locomotion detects differences in recovery after spinal cord injury in five common mouse strains. J Neurotrauma 23: 635-659.[Crossref]

12. Hill RL, Zhang YP, Burke DA, Devries WH, Zhang Y, et al. (2009) Anatomical and Functional Outcomes following a Precise, Graded, Dorsal Laceration Spinal Cord Injury in C57BL/6 Mice. J Neurotrauma 26: 1-15.[Crossref]

13. Nishimura S, Yasuda A, Iwai H, Takano M, Kobayashi Y, et al. (2013) Time-dependent changes in the microenvironment of injured spinal cord affects the therapeutic potential of neural stem cell transplantation for spinal cord injury. Mol Brain 6: 3.[Crossref]

14. Luppi PH, Fort P, Jouvet M (1990) Iontophoretic application of unconjugated cholera toxin $\mathrm{B}$ subunit $(\mathrm{CTb})$ combined with immunohistochemistry of neurochemical substances: a method for transmitter identification of retrogradely labeled neurons. Brain Res 534: 209-224.[Crossref]

Copyright: (C2016 Arimitsu N. This is an open-access article distributed under the terms of the Creative Commons Attribution License, which permits unrestricted use, distribution, and reproduction in any medium, provided the original author and source are credited. 